\title{
Exponential stability of stochastic functional differential equations with Markovian switching and delayed impulses via Razumikhin method
}

Lijun $\operatorname{Pan}^{1,2}$ and Jinde Cao ${ }^{1^{*}}$

\author{
* Correspondence: jdcao@seu.edu. \\ cn \\ ${ }^{1}$ Department of Mathematics, \\ Southeast University, Nanjing \\ 210096, P. R. China \\ Full list of author information is \\ available at the end of the article
}

\begin{abstract}
In this article, by using Razumikhin-type technique, we investigate pth moment exponential stability of stochastic functional differential equations with Markovian switching and delayed impulses. Several stability theorems of impulsive hybrid stochastic functional differential equations are derived. It is assumed that the state variables on the impulses can relate to the finite delay. These new results are employed to a class of $n$-dimensional linear impulsive hybrid stochastic systems with bounded time-varying delay. Moreover, an effective $M$-matrix method is introduced to study the exponential stability of these hybrid systems. Meanwhile, some examples and simulations are given to show our results.
\end{abstract}

Keywords: Razumikhin theorem, stochastic functional differential equations, impulse, M-matrix, Markovian chain

\section{Introduction}

Stochastic differential equation is an emerging field drawing attention from both theoretical and applied disciplines, which has been successfully applied to problems in mechanical, electrical, economics, physics and several fields in engineering. For details, see [1-6] and the references therein. Recently, stability of stochastic differential equations with Markovian switching has received a lot of attention [7-12]. For example, Ji and Chizeck [7] and Mariton [8] studied the stability of a jump linear equation

$$
d x(t)=A(r(t)) x(t) d t
$$

where $x(t)$ takes values in $R^{n}, r(t)$ is a Markovian chain taking values in $S=\{1,2, \ldots$, $N\}$. Mao [9] discussed the stability of nonlinear stochastic differential equation with Markovian switching of the form

$$
d x(t)=f(x(t), t, r(t)) d t+g(x(t), t, r(t)) d \omega(t) .
$$

In [10], Mao studied the stability of stochastic functional differential equation with Markovian switching of the form

$$
d x(t)=f\left(x_{t}, t, r(t)\right) d t+g\left(x_{t}, t, r(t)\right) d \omega(t)
$$

Impulsive effects are common phenomena due to instantaneous perturbations at certain moment, such phenomena are described by impulsive differential equation which 
have been used effciently in modelling many practical problems that arise in the fields of engineering, physics, and science as well. So the theory of impulsive differential equations is also attracting much attention in recent years [13-19]. Correspondingly, a lot of stability results of impulsive stochastic functional differential equations have been obtained [20-26]. However, there are few results on the stability of impulsive stochastic differential equation with Markovian switching. In [27], Wu and Sun established some stability criteria of $p$-moment stability for stochastic differential equations with impulsive jump and Markovian switching.

In this article, we shall extend Razumikhin method $[10,12]$ to investigate the $p$ th moment exponential stability of the following stochastic functional differential equations with Markovian switching and delayed impulse

$$
\left\{\begin{array}{l}
d x(t)=f\left(x_{t}, t, r(t)\right) d t+g\left(x_{t}, t, r(t)\right) d \omega(t) \quad t \geq 0, \quad t \neq t_{k} \\
\Delta x\left(t_{k}\right)=I_{k}\left(x\left(t_{k}\right), x_{t_{k}}, t_{k}, r\left(t_{k}\right)\right) \quad, k=1,2, \ldots, \\
x(t)=\xi, \quad t \in[-\tau, 0] .
\end{array}\right.
$$

The state variables on the impulses relate to the finite delay, which implies that the impulsive effects are more general than those given in $[20,22,23]$. Some Theorems on the $p$ th moment exponential stability are derived in the case that the impulsive gain $d_{i k}+\bar{d}_{i k}<1$ or $d_{i k}+\bar{d}_{i k} \geq 1$. These new results are employed to the $n$-dimensional impulsive hybrid stochastic systems with bounded time-varying delay. Useful criteria in terms of an $M$-matrix (see Berman and Plemmons [28]) which can be verified much more easily are established. Meanwhile, examples and simulations are provided to show the impulsive effects play an important role in the stability for hybrid stochastic systems. The rest of this article is organized as follows. In Section 2, stochastic functional differential equations with Markovian switching and delayed impulses together with some definitions of $p$ th moment exponential stability are presented. In Section 3, the Razumikhin-type theorems on $p$ th moment exponential stability for stochastic functional differential equations with Markovian switching and delayed impulses are established. In Section 4, these results will then be applied to the $n$ dimensional hybrid stochastic delay systems and $M$-matrix method is introduced to verify the stability easily. Finally, examples are given to demonstrate our effective results in Section 5.

\section{Preliminaries}

Let $R=(-\infty,+\infty), R^{+}=[0,+\infty), R^{n}$ denote the $n$-dimensional Euclidean space with the Euclidean norm $|\cdot|$. If $A$ is a vector or matrix, its transpose is denoted by $A^{T}$, and its norm is denoted by $\|A\|=\sqrt{\lambda_{\max }\left(A^{T} A\right)}$, where $\lambda_{\max }(\cdot)$ is the maximum eigenvalue of a matrix. $\omega(t)=\left(\omega_{1}(t), \omega_{2}(t), \ldots, \omega_{m}(t)\right)^{T}$ is an $m$-dimensional Brownian motion on a complete probability space $(\Omega, \mathscr{F}, P)$ with a natural filtration $\left\{\mathscr{F}_{t}\right\}_{t \geq 0}$ satisfying the usual conditions, (i.e. $\mathscr{F}_{t}=\sigma\{\omega(s): 0 \leq s \leq t\}$ ).

Let $\tau>0$ and $P C\left([-\tau, 0], R^{n}\right)=\left\{\psi:[-\tau, 0] \rightarrow R^{n} \mid \psi\left(t^{+}\right), \psi(t)\right.$ exist, and $\left.\psi\left(t^{-}\right)=\psi(t)\right\}$ with the norm $\|\psi\|=\sup _{-\tau \leq \theta \leq 0}|\psi(\theta)|$, where $\psi\left(t^{+}\right)$and $\psi\left(t^{-}\right)$denote the right-hand and left-hand limits of function $\psi(t)$ at $t$.

Denoted by $P C_{\mathscr{F}_{0}}^{b}\left([-\tau, 0] ; R^{n}\right)$ the family of all bounded, $\mathscr{F}_{0}$ - measurable, $P C([-\tau$, $0] ; R^{n}$ )-valued random variables. For $p>0$, denoted by $P C_{\mathscr{F}_{t}}^{p}\left([-\tau, 0], R^{n}\right)$ the family of 
all $\mathscr{F}_{t}$-measurable $P C\left([-\tau, 0], R^{n}\right)$-valued random variables $\psi$ such that $\int_{-\tau}^{0} E|\psi(\theta)|^{p} d \theta<\infty$. Let $r(t)(t>0)$ be a right-continuous Markovian chain on the probability space taking values in a finite state space $S=\{1,2, \ldots, N\}$ with generator $\Gamma=\left(\gamma_{i j}\right)_{N \times N}$ given by

$$
P\{r(t+\Delta)=j \mid r(t)=i\}=\left\{\begin{array}{l}
\gamma_{i j} \Delta+o(\Delta), \text { if } i \neq j, \\
1+\gamma_{i j} \Delta+o(\Delta), \text { if } i=j,
\end{array}\right.
$$

where $\Delta>0$. Here $\gamma_{i j} \geq 0$ is the transition rate from $i$ to $j$ while

$$
\gamma_{i i}=-\sum_{i \neq j} \gamma_{i j}
$$

We assume that the Markovian chain $r(t)$ is independent of the Brownian motion $\omega(t)$. It is known that almost every sample path of $r(t)$ is a right-continuous step function with a finite number of simple jumps in any finite subinterval of $R^{+}$.

Consider the following impulsive hybrid stochastic functional differential equation of the form

$$
\left\{\begin{array}{l}
d x(t)=f\left(x_{t}, t, r(t)\right) d t+g\left(x_{t}, t, r(t)\right) d \omega(t), \quad t \geq 0, \quad t \neq t_{k} \\
\Delta x\left(t_{k}\right)=I_{k}\left(x\left(t_{k}\right), x_{t_{k}}, t_{k}, r\left(t_{k}\right)\right), \quad k=1,2, \ldots, \\
x(t)=\xi, t \in[-\tau, 0],
\end{array}\right.
$$

where

$$
\xi \in P C_{\mathscr{F}_{0}}^{b}\left([-\tau, 0] ; R^{n}\right), x(t)=\left(x_{1}(t), x_{2}(t), \ldots, x_{n}(t)\right)^{T}
$$

$x\left(t_{k}^{+}\right)=\lim _{h \rightarrow 0+} x\left(t_{k}+h\right), x\left(t_{k}\right)=\lim _{h \rightarrow 0-} x\left(t_{k}+h\right), t_{k} \geq 0$,

$x\left(t_{k}^{+}\right)=\lim _{h \rightarrow 0+} x\left(t_{k}+h\right), x\left(t_{k}\right)=\lim _{h \rightarrow 0-} x\left(t_{k}+h\right), t_{k} \geq 0$ are impulsive moments satisfying $t_{k}$ $<t_{k+1}$ and $\lim _{k \rightarrow+\infty} t_{k}=+\infty, \Delta x\left(t_{k}\right)=x\left(t_{k}^{+}\right)-x\left(t_{k}\right)$ represents the jump in the state $x$ at $t_{k}$ with $I_{k}$ determining the size of the jump, $f: P C\left([-\tau, 0] ; R^{n}\right) \times R^{+} \times S \rightarrow R^{n}, g: P C(-\tau, 0]$; $\left.R^{n}\right) \times R^{+} \times S \rightarrow R^{n \times m}, I_{k}: R^{n} \times P C\left([-\tau, 0] ; R^{n}\right) \times R^{+} \times S \rightarrow R^{n}$.

Throughout this article, we assume that $f, g$ and $I_{k}$ satisfy the necessary conditions for the global existence and uniqueness of solutions for all $t \geq 0$. For any $\xi \in P C_{\mathscr{F}_{0}}^{b}\left([-\tau, 0] ; R^{n}\right)$, there exists a unique stochastic process satisfying Equation (2.1) denoted by $x(t ; \xi)$, which is continuous on the left-hand side and limitable on the right-hand side. Also we assume that $f(0, t, i) \equiv 0, g(0, t, i) \equiv 0$ and $I_{k}(0,0, t, i) \equiv 0, k=$ $1,2, \ldots$, which implies that $x(t) \equiv 0$ is an equilibrium solution.

Let $\mathscr{C}_{1}^{2}\left(R^{n} \times[-\tau, \infty) \times S ; R^{+}\right)$be the family of all nonnegative functions $V(x, t, i)$ on $R^{n} \times[-\tau, \infty) \times S$ which are continuous on $R^{n} \times\left(t_{k-1}, t_{k}\right] \times S, V_{t}, V_{x}, V_{x x}$ are continuous on $R^{n} \times\left(t_{k-1}, t_{k}\right] \times S$. For each $V \in \mathscr{C}_{1}^{2}\left(R^{n} \times[-\tau, \infty) \times S ; R^{+}\right)$, we define an operator $\mathscr{L} V: P C_{\mathscr{F}_{t}}^{b}\left([-\tau, 0] ; R^{n}\right) \times\left(t_{k-1}, t_{k}\right] \times S \rightarrow R$ associated with Equation (2.1) as follows:

$$
\begin{aligned}
& \mathscr{L} V(\phi, t, i)=V_{t}(\phi(0), t, i)+V_{x}(\phi(0), t, i) f(\phi, t, i) \\
& +\frac{1}{2} \operatorname{trace}\left[g^{T}(\phi, t, i) V_{x x}(\phi(0), t, i) g(\phi, t, i)\right]+\sum_{j=1}^{N} \gamma_{i j} V(\phi(0), t, j),
\end{aligned}
$$


where

$$
\begin{aligned}
& V_{t}(x, \quad t, \quad i)=\frac{\partial V(x, t, i)}{\partial t}, V_{x}(x, t, i)=\left(\frac{\partial V(x, t, i)}{\partial x_{1}}, \ldots, \frac{\partial V(x, t, i)}{\partial x_{n}}\right), V_{x x}(x, t, i)= \\
& \left(\frac{\partial^{2} V(x, t, i)}{\partial x_{i} x_{j}}\right)_{n \times n} .
\end{aligned}
$$

Definition 2.1. The zero solution of Equation (2.1) are said to be $p$ th moment exponentially stable if there exists $\eta>0$ such that for any initial values $\xi \in P C_{\mathscr{F}_{0}}^{b}\left([-\tau, 0] ; R^{n}\right)$ and $t \geq 0$

$$
\limsup _{t \rightarrow \infty} \frac{1}{t} \log \left(E|x(t ; \xi)|^{p}\right) \leq-\eta \text {. }
$$

Remark 2.1. When $p=2$, it is often called to be exponentially stable in mean square.

\section{Stability analysis}

In the following, we shall establish some criteria on $p$ th moment exponential stability for Equation (2.1).

Theorem 3.1. Assume that $V \in \mathscr{C}_{1}^{2}\left(R^{n} \times[-\tau, \infty) \times S ; R^{+}\right)$and constants $p>0, c_{1}>$ $0, c_{2}>0, d_{i k} \geq 0, \bar{d}_{i k} \geq 0, d_{i k}^{2}+\bar{d}_{i k}^{2} \neq 0, \delta>0, \lambda>0, \eta_{i} \geq 0, i \in S, k=1,2, \ldots$ such that

(i) $c_{1}|x|^{p} \leq V(x, t, i) \leq c_{2}|x|^{p}$ for all $(t, x) \in R^{n} \times[-\tau, \infty) \times S$;

(ii) for all $t \in\left(t_{k-1}, t_{k}\right]$ and $i \in S, E \mathscr{L} V(\phi, t, i) \leq \eta_{i} \operatorname{EV}(\phi(0), t, i)$ whenever $E\left[\min _{1 \leq i \leq N} V(\phi(\theta), t+\theta, i)\right]<q e^{\lambda \tau} E\left[\max _{1 \leq i \leq N} E V(\phi(0), t, i)\right]$;

$$
\text { for all }
$$

$i \in S$,

$E V\left(\phi(0)+I_{k}\left(\phi(0), \phi(\theta), t_{k}, i\right), t_{k}^{+}, i\right) \leq d_{i k} E V\left(\phi(0), t_{k}, i\right)+\bar{d}_{i k} \sup _{-\tau \leq \theta<0} E V\left(\phi(\theta), t_{k}, i\right) ;$

(iv) $\sup _{1 \leq k<+\infty}\left\{t_{k}-t_{k-1}\right\} \leq \delta$;

(v) for any $i \in S, \lambda+\eta_{i} \leq \frac{\ln q}{\delta}$,

then the zero solution of Equation (2.1) is $p$ th moment exponentially stable with $p$ th moment exponent $\lambda$, where $\phi=\{\phi(\theta) \mid-\tau \leq \theta \leq 0\} \in P C_{\mathscr{F}_{t}}^{p}\left([-\tau, 0] ; R^{n}\right), q=\frac{1}{\max _{i \in S, 1 \leq k<+\infty}\left\{d_{i k}+\bar{d}_{i k} e^{\lambda \tau}\right\}}>1$.

Proof. For any $\xi \in P C_{\mathscr{F}_{0}}^{b}\left([-\tau, 0] ; R^{n}\right)$, we denote the solution $x(t)=x(t ; \xi)$ of $(2.1)$ and extend $r(t)=r(0)=r_{0}$ for all $t \in[-\tau, 0]$. Let $\varepsilon$ be small enough such that $t+\varepsilon \in$ $\left(t_{k-1}, t_{k}\right)$. By generalized Itô formula, we have

$$
E V(x(t+\varepsilon), t+\varepsilon, r(t+\varepsilon))=E V(x(t), t, r(t))+\int_{t}^{t+\varepsilon} E \mathscr{L} V(x(s), s, r(s)) d s .
$$

Let $\varepsilon \rightarrow 0$, it follows that for $t \in\left(t_{k-1}, t_{k}\right]$

$$
D^{+} E V(x(t), t, r(t))=E \mathscr{L} V(x(t), t, r(t)) .
$$

Let $W(t)=e^{\lambda t} E V(x(t), t, i)$, we have for $t \in\left(t_{k-1}, t_{k}\right]$

$$
D^{+} W(t)=\lambda e^{\lambda t} E V(x(t), t, i)+e^{\lambda t} D^{+} E \mathscr{L} V(x(t), t, i) .
$$


From (iii), we have

$$
W\left(t_{k}^{+}\right)=e^{\lambda t_{k}} E V\left(x\left(t_{k}^{+}\right), t_{k}^{+}, i\right) \leq d_{i k} W\left(t_{k}\right)+\bar{d}_{i k} e^{\lambda \tau} \sup _{-\tau \leq \theta \leq 0} W\left(t_{k}+\theta\right) .
$$

Taking $M>0$ such that

$$
\sup _{-\tau \leq \theta \leq 0} W(\theta)<\frac{M}{q}
$$

we can claim that for $t \geq-\tau$

$$
W(t)<M \text {. }
$$

It is easy to see that $W(t)<M$ for $t \in[-\tau, 0]$. Now, we shall prove that

$$
W(t)<M, t \in\left(0, t_{1}\right] .
$$

Otherwise, there exists a $t^{*} \in\left(0, t_{1}\right]$ such that

$$
W\left(t^{*}\right)=M, W(t)<M,-\tau<t<t^{*} .
$$

In view of the continuity of $W(t)$ in $\left[0, t_{1}\right]$, there exists a $t^{* *} \in\left(0, t^{*}\right)$ such that

$$
W\left(t^{* *}\right)=\frac{M}{q}, W(t)>\frac{M}{q}, t \in\left(t^{* *}, t^{*}\right] .
$$

For $t \in\left[t^{* * *}, t^{*}\right], \theta \in[-\tau, 0]$, we have

$$
q W(t)>W(t+\theta) .
$$

Then we obtain

$$
\begin{aligned}
q e^{\lambda \tau}\left[\max _{1 \leq i \leq N} E V(x(t), t, i)\right] & \geq q e^{-\lambda(t-\tau)} W(t)>e^{-\lambda(t-\tau)} W(t+\theta) \\
& \geq \min _{1 \leq i \leq N} E V(x(t+\theta), t+\theta, i) .
\end{aligned}
$$

Together with (3.3) and (ii), for $t \in\left[t^{* *}, t^{*}\right]$, we have

$$
\begin{aligned}
D^{+} W(t) & \leq \lambda e^{\lambda t} E V(x(t), t, i)+\eta_{i} e^{\lambda t} E V(x(t), t, i) \\
& =\left(\lambda+\eta_{i}\right) e^{\lambda t} E V(x(t), t, i)=\left(\lambda+\eta_{i}\right) W(t) .
\end{aligned}
$$

Thus

$$
M=W\left(t^{*}\right) \leq W\left(t^{* *}\right) e^{\left(\lambda+\eta_{i}\right)\left(t^{*}-t^{* *}\right)}=\frac{M}{q} e^{\left(\lambda+\eta_{i}\right)\left(t^{*}-t^{* *}\right)}<\frac{M}{q} e^{\left(\lambda+\eta_{i}\right) t_{1}} \leq M .
$$

This is a contradiction. Hence (3.7) holds. From (iii), we obtain

$$
W\left(t_{1}^{+}\right) \leq d_{i 1} W\left(t_{1}\right)+\bar{d}_{i 1} e^{\lambda \tau} \sup _{-\tau \leq \theta \leq 0} W\left(t_{1}+\theta\right)<\frac{M}{q}<M .
$$

Next, we shall show that

$$
W(t)<M, \quad t \in\left(t_{1}, t_{2}\right] .
$$

If it does not hold, there exists a $t_{1}^{*} \in\left(t_{1}, t_{2}\right]$ such that

$$
W\left(t_{1}^{*}\right)=M, W(t)<M, \quad t \in\left[-\tau, t_{1}^{*}\right] .
$$


In view of the continuity of $W(t)$ in $\left(t_{1}, t_{2}\right]$, there exists a $t_{1}^{* *} \in\left(t_{1}, t_{1}^{*}\right)$ such that

$$
W\left(t_{1}^{* *}\right)=\frac{M}{q}, W(t)>\frac{M}{q}, \quad t \in\left(t_{1}^{* *}, t_{1}^{*}\right] .
$$

For $t \in\left[t_{1}^{* *}, t_{1}^{*}\right], \theta \in[-\tau, 0]$, we have $q W(t)>W(t+\theta)$. It follows from (3.3) and (ii) that for $t \in\left[t_{1}^{* *}, t_{1}^{*}\right]$

$$
D^{+} W(t) \leq\left(\lambda+\eta_{i}\right) W(t) .
$$

Then, we have

$$
M=W\left(t_{1}^{*}\right) \leq W\left(t_{1}^{* *}\right) e^{\left(\lambda+\eta_{i}\right)\left(t_{1}^{*}-t_{1}^{* *}\right)}=\frac{M}{q} e^{\left(\lambda+\eta_{i}\right)\left(t_{1}^{*}-t_{1}^{* *}\right)}<\frac{M}{q} e^{\left(\lambda+\eta_{i}\right)\left(t_{2}-t_{1}\right)} \leq M ，
$$

which is a contradiction. Thus, (3.15) holds. By induction, we can prove that for $k=$ $1,2, \ldots$

$$
W(t)<M, \quad t \in\left(t_{k-1}, t_{k}\right]
$$

Therefore, we have for $t \geq-\tau$

$$
W(t)<M
$$

From (i) and the above inequality, we have

$$
c_{1} E|x(t)|^{p} \leq E V(t) \leq M e^{-\lambda t},
$$

which implies that

$$
E|x(t)|^{p} \leq \frac{M}{c_{1}} e^{-\lambda t}
$$

The proof of Theorem 3.1 is complete.

Remark 3.1. In Theorem 3.1, the zero solution of hybrid stochastic functional differential equations without impulses is allowed to be unstable. In this case, the delayed impulses are key in stabilizing the hybrid stochastic equations. It requires the nearest impulse time interval must be sufficiently small and the maximal impulsive gain $\max _{i \in S, 1 \leq k<+\infty}\left\{d_{i k}+\bar{d}_{i k}\right\}<1$.

Theorem 3.2. Assume that $V \in \mathscr{C}_{1}^{2}\left(R^{n} \times[-\tau, \infty) \times S ; R^{+}\right)$and constants $p>0, c_{1}>$ $0, c_{2}>0, d_{i k} \geq 0, \bar{d}_{i k} \geq 0, d_{i k}^{2}+\bar{d}_{i k}^{2} \neq 0, \gamma_{i}>0, \mu>0, \lambda>0, i \in S, k=1,2, \ldots$ such that

(i) $c_{1}|x|^{p} \leq V(t, x) \leq c_{2}|x|^{p}$ for all $(t, x) \in[-\tau, \infty) \times R^{n}$;

(ii) for all $t \in\left(t_{k-1}, t_{k}\right]$ and $i \in S, \varepsilon>0 \operatorname{ELV}(\phi, t, i) \leq-\gamma_{i} E V(\phi(0), t, i)$ whenever $E\left[\min _{1 \leq i \leq N} V(\phi(\theta), t+\theta, i)\right] \leq q e^{\lambda \tau} E\left[\max _{1 \leq i \leq N} E V(\phi(0), t, i)\right]$

(iii) for all $i \quad \in \quad S$, $E V\left(\phi(0)+I_{k}\left(\phi(0), \phi(\theta), t_{k}, i\right), t_{k}^{+}, i\right) \leq d_{i k} E V\left(\phi(0), t_{k}, i\right)+\bar{d}_{i k} \sup _{-\tau \leq \theta \leq 0} E V\left(\phi(\theta), t_{k}, i\right) ;$

(iv) $\inf _{1 \leq k<+\infty}\left\{t_{k}-t_{k-1}\right\} \geq \mu$;

(v) for any $i \in S, \gamma_{i}-\lambda>\frac{\ln q}{\mu}$,

then the zero solution of Equation (2.1) is $p$ th moment exponentially stable, where $\phi=\{\phi(\theta) \mid-\tau \leq \theta \leq 0\} \in P C_{\mathscr{F}_{t}}^{p}\left([-\tau, 0] ; R^{n}\right), q=\frac{1}{\max _{i \in S, 1 \leq k<+\infty}\left\{d_{i k}+\bar{d}_{i k} e^{\lambda \tau}\right\}}>1$. 
Proof. Since $(v)$ holds, we can choose sufficiently small $\varepsilon>0$ such that $\gamma_{i}-\lambda \geq \frac{\ln (q+\varepsilon)}{\mu}$ for all $i \in S$. Let $W(t)=e^{\lambda t} E V(x(t), t, i)$, we have for $t \in\left(t_{k-1}, t_{k}\right]$

$$
D^{+} W(t)=\lambda e^{\lambda t} E V(x(t), t, i)+e^{\lambda t} D^{+} E \mathscr{L} V(x(t), t, i) .
$$

From (iii), we have

$$
W\left(t_{k}^{+}\right)=e^{\lambda t_{k}} E V\left(x\left(t_{k}^{+}\right), t_{k}^{+}, i\right) \leq d_{i k} W\left(t_{k}\right)+\bar{d}_{i k} e^{\lambda \tau} \sup _{-\tau \leq \theta \leq 0} W\left(t_{k}+\theta\right) .
$$

Taking $M>0$ such that

$$
\sup _{-\tau \leq \theta \leq 0} W(\theta)<\frac{M}{q+\varepsilon}
$$

we shall show that for $\mathrm{t} \geq-\tau$

$$
W(t)<M \text {. }
$$

It is easy to see that $W(t)<M$ for $t \in[-\tau, 0]$. Now, we shall prove that

$$
W(t)<M, t \in\left(0, t_{1}\right] .
$$

If it does not hold, there exists a $t^{*} \in\left(0, t_{1}\right]$ such that

$$
W\left(t^{*}\right)=M, W(t)<M,-\tau<t<t^{*} .
$$

In view of the continuity of $W(t)$ in $\left[0, t_{1}\right]$, there exists a $t^{* *} \in\left[0, t^{*}\right)$ such that

$$
W\left(t^{* *}\right)=\frac{M}{q+\varepsilon}, W(t)>\frac{M}{q+\varepsilon}, \quad t \in\left(t^{* *}, t^{*}\right] .
$$

For $t \in\left[t_{\varepsilon}^{* *}, t^{*}\right], \theta \in[-\tau, 0]$

$$
(q+\varepsilon) W(t)>W(t+\theta) .
$$

Then

$$
(q+\varepsilon) e^{\lambda \tau}\left[\max _{1 \leq i \leq N} E V(x(t), t, i)\right] \geq \min _{1 \leq i \leq N} E V(x(t+\theta), t+\theta, i) .
$$

Together with (3.24) and (ii), for $t \in\left[t^{* *}, t^{*}\right]$, we have

$$
D^{+} W(t) \leq \lambda e^{\lambda t} E V(x(t), t, i)+e^{\lambda t} E \mathscr{L} V(x(t), t, i) \leq\left(\lambda-\gamma_{i}\right) W(t) \leq 0 .
$$

Thus

$$
M=W\left(t^{*}\right) \leq W\left(t^{* *}\right)=\frac{M}{q+\varepsilon}<M .
$$

This is a contradiction. Next, we shall show that

$$
W\left(t_{1}\right) \leq \frac{M}{q+\varepsilon} .
$$

If it does not hold, we have

$$
W\left(t_{1}\right)>\frac{M}{q+\varepsilon} .
$$


Since the continuity of $W\left((t)\right.$ in $\left[0, t_{1}\right]$, there exists a $\bar{t} \in\left[0, t_{1}\right)$ such that

$$
W\left(\bar{t}_{\varepsilon}\right)=\frac{M}{q+\varepsilon}, W(t)>\frac{M}{q+\varepsilon}, t \in\left(\bar{t}, t_{1}\right]
$$

For $t \in\left[\bar{t}, t_{1}\right], \theta \in[-\tau, 0]$, we have

$$
(q+\varepsilon) W(t)>W(t+\theta) \text {. }
$$

By (3.24) and (ii), for $t \in\left[\bar{t}, t_{1}\right], \theta \in[-\tau, 0]$, we have

$$
D^{+} W(t) \leq\left(\lambda-\gamma_{i}\right) W(t) \leq 0
$$

Thus

$$
W\left(t_{1}\right) \leq W(\bar{t})=\frac{M}{q+\varepsilon}
$$

which is a contradiction. It follows from (3.25), (3.35) and (3.28) that

$$
W\left(t_{1}^{+}\right) \leq d_{i 1} W\left(t_{1}\right)+\bar{d}_{i 1} e^{\lambda \tau} \sup _{-\tau \leq \theta \leq 0} W\left(t_{1}+\theta\right) \leq \frac{\left(d_{i 1}+\bar{d}_{i 1} e^{\lambda \tau}\right) M}{q+\varepsilon}<M .
$$

Furthermore, we can prove that

$$
W(t)<M, \quad t \in\left(t_{1}, t_{2}\right] .
$$

Indeed, there exists a $\bar{t}_{1} \in\left(t_{1}, t_{2}\right]$ such that

$$
W\left(\bar{t}_{1}\right)=M, W(t)<M, t \in\left[-\tau, \bar{t}_{1}\right)
$$

If $W(t)>\frac{M}{q+\varepsilon}$ for $t \in\left(t_{1}, \bar{t}_{1}\right)$. Then $(q+\varepsilon) W(t)>W(t+\theta)$ for $t \in\left(t_{1}, \bar{t}_{1}\right), \theta \in[-\tau, 0]$. Thus by (3.24) and (ii), for $t \in\left(t_{1}, \bar{t}_{1}\right)$, we have $D^{+} W(t) \leq(\lambda-\gamma) W(t) \leq 0$. It follows that

$$
W\left(\bar{t}_{1}\right) \leq W\left(t_{1}^{+}\right)<M
$$

This is a contradiction. If there exists a $\overline{\bar{t}}_{1} \in\left(t_{1}, \bar{t}_{1}\right)$ such that

$$
W\left(\overline{\bar{t}}_{1}\right) \leq \frac{M}{q+\varepsilon}
$$

In view of the continuity of $W(t)$ in $\left(t_{1}, t_{2}\right]$, there exists a $\overline{\bar{t}}_{1}^{\prime} \in\left[\overline{\bar{t}}_{1}, \bar{t}_{1}\right)$ such that

$$
W\left(\overline{\bar{t}}_{1}^{\prime}\right)=\frac{M}{q+\varepsilon}, W(t)>\frac{M}{q+\varepsilon}, t \in\left(\overline{\bar{t}}_{1}^{\prime}, \bar{t}_{1}\right]
$$

Then for $t \in\left[\overline{\bar{t}}_{1}^{\prime}, \bar{t}_{1}\right], \quad \theta \in[-\tau, 0], \quad(q+\varepsilon) W(t)>W(t+\theta)$. Thus for $W\left(\bar{t}_{1}\right) \leq W\left(\overline{\bar{t}}_{1}^{\prime}\right)=\frac{M}{q+\varepsilon}<M, D^{+} W(t) \leq(\lambda-\gamma) W(t) \leq 0$. It follows that

$$
W\left(\bar{t}_{1}\right) \leq W\left(\overline{\bar{t}}_{1}^{\prime}\right)=\frac{M}{q+\varepsilon}<M,
$$

which leads to a contradiction. Moreover, we can conclude that $W\left(t_{2}\right) \leq \frac{M}{q+\varepsilon}$. If this does not hold, we have $W\left(t_{2}\right)>\frac{M}{q+\varepsilon}$. To prove the conclusion, two cases are to be considered.

Case $(i)$. For $t \in\left(t_{1}, t_{2}\right], W(t)>\frac{M}{q+\varepsilon}$. From (3.26), (3.28) and (3.43), we have for $t \in$ $\left(t_{1}, t_{2}\right], \theta \in[-\tau, 0]$ 


$$
(q+\varepsilon) W(t)>W(t+\theta) .
$$

Then by (3.24) and (ii), for $t \in\left(t_{1}, t_{2}\right]$

$$
D^{+} W(t) \leq\left(\lambda-\gamma_{i}\right) W(t) .
$$

Thus

$$
W\left(t_{2}\right) \leq W\left(t_{1}^{+}\right) e^{\left(\lambda-\gamma_{i}\right)\left(t_{2}-t_{1}\right)}<M e^{\left(\lambda-\gamma_{i}\right)\left(t_{2}-t_{1}\right)} \leq \frac{M}{q+\varepsilon},
$$

which leads to a contradiction.

Case $(i i)$. There exists a $\tilde{t}_{1 \varepsilon} \in\left(t_{1}, t_{2}\right]$ such that $W\left(\tilde{t}_{1}\right) \leq \frac{M}{q+\varepsilon}$. Since $W\left(t_{2}\right)>\frac{M}{q+\varepsilon}$ and in view of the continuity of $W(t)$ in $\left(t_{1}, t_{2}\right]$, there exists a $\tilde{t}_{1}^{*} \in\left[\tilde{t}_{1}, t_{2}\right]$ such that

$$
W\left(\tilde{t}_{1}^{*}\right)=\frac{M}{q+\varepsilon}, W(t)>\frac{M}{q+\varepsilon}, \quad t \in\left(\tilde{t}_{1}^{*}, t_{2}\right] .
$$

For $t \in\left(\tilde{t}_{1}^{*}, t_{2}\right], \theta \in[-\tau, 0]$, we have

$$
(q+\varepsilon) W(t)>W(t+\theta) \text {. }
$$

It follows from (3.24) and (ii) that for $t \in\left(\tilde{t}_{1}^{*}, t_{2}\right]$

$$
D^{+} W(t) \leq\left(\lambda-\gamma_{i}\right) W(t) \leq 0
$$

Thus

$$
W\left(t_{2}\right) \leq W\left(\tilde{t}_{1}^{*}\right)=\frac{M}{q} .
$$

This is a contradiction. By induction, we can prove that for $k=1,2, \ldots$

$$
W(t)<M, t \in\left(t_{k-1}, t_{k}\right] .
$$

Therefore, we have for $t \geq-\tau$

$$
W(t)<M \text {. }
$$

The proof of Theorem 3.1 is complete.

Remark 3.2. In Theorem 3.2, it is permitted that the maximal impulsive gain $\max _{i \in S, 1 \leq k<+\infty}\left\{d_{i k}+\bar{d}_{i k}\right\} \geq 1$. This means that the hybrid stochastic equation not only can achieve exponential stability but also is exponential stability with delayed impulses. In this case, it requires that the minimal impulse time interval must be sufficiently large such that the hybrid stochastic differential equations with delayed impulses can make keep its stability property.

\section{Some consequences}

In the following, we shall apply the above new results to a class of linear impulsive hybrid stochastic systems by using Lyapunov function and $M$-matrix method.

Consider the following $n$-dimensional impulsive hybrid stochastic delay differential equation 


$$
\left\{\begin{array}{l}
d x(t)=[A(r(t)) x(t)+B(r(t)) x(t-\tau(t))] d t \\
\quad+[C(r(t)) x(t)+D(r(t)) x(t-\tau(t))] d \omega(t), \quad t \geq 0, \quad t \neq t_{k} \\
\Delta x\left(t_{k}\right)=I_{k}\left(x\left(t_{k}\right), x\left(t_{k}-\tau\left(t_{k}\right)\right), t_{k}, r\left(t_{k}\right)\right), k=1,2, \ldots \\
x(t)=\xi, t \in[-\tau, 0]
\end{array}\right.
$$

where $0 \leq \tau(t) \leq \tau$ is continuous, $\tau$ is a positive constant. For convenience, we denote $A(r(t))=A_{i}, B(r(t))=B_{i}, C(r(t))=C_{i}, D(r(t))=D_{i}$, where $A_{i}=\left(a_{u v}(i)\right)_{n \times n}, B_{i}=\left(b_{u v}(i)\right)$ ${ }_{n \times n}, C_{i}=\left(c_{u v}(i)\right)_{n \times n}, D_{i}=\left(d_{u v}(i)\right)_{n \times n}$.

Theorem 4.1. Assume that there exist symmetric positive definite matrices $Q_{i}$ and constants $d_{i k} \geq 0, \bar{d}_{i k} \geq 0, d_{i k}^{2}+\bar{d}_{i k}^{2} \neq 0, \delta>0, \lambda>0, \rho_{i}>0, \sigma_{i}>0, \bar{\eta}_{i} \geq 0, \eta_{i}, i \in S, k=1,2, \ldots$ such that

(i) for all $i \in S$

$$
\Gamma_{i}=Q_{i} A_{i}+A_{i}^{T} Q_{i}+\rho_{i} Q_{i}^{2}+C_{i}^{T} Q_{i} C_{i}+\sigma_{i} C_{i}^{T} C_{i}+\sum_{j=1}^{N} \gamma_{i j} Q_{j}-\eta_{i} Q \leq 0,
$$

and

$$
\bar{\Gamma}_{i}=\rho_{i}^{-1} B_{i}^{T} B_{i}+\sigma_{i}^{-1} D_{i}^{T} Q_{i}^{2} D_{i}+D_{i}^{T} Q_{i} D_{i}-\bar{\eta}_{i} Q_{i} \leq 0,
$$

where $\Gamma_{i}, \bar{\Gamma}_{i} \leq 0,0 \leq i \leq N$ mean that matrices $\Gamma_{i}, \bar{\Gamma}_{i}$ are negative semi-definite; (ii) for all $i \in S$

$$
\begin{aligned}
& \begin{array}{l}
E\left[\left(x\left(t_{k}+I_{k}\left(x\left(t_{k}\right), x\left(t_{k}-\tau\left(t_{k}\right)\right), t_{k}, i\right)\right)^{T} Q_{i}\left(x\left(t_{k}+I_{k}\left(x\left(t_{k}\right), x\left(t_{k}-\tau\left(t_{k}\right)\right), t_{k}, i\right)\right)\right]\right.\right. \\
\quad \leq d_{i k} E\left[x^{T}\left(t_{k}\right) Q_{i} x\left(t_{k}\right)\right]+d_{i k} E\left[x^{T}\left(t_{k}-\tau\left(t_{k}\right)\right) Q_{i} x\left(t_{k}-\tau\left(t_{k}\right)\right)\right] ;
\end{array} \\
& \text { (iii) } \sup _{1 \leq k<+\infty}\left\{t_{k}-t_{k-1}\right\} \leq \delta_{;} \\
& \text {(iv) for all } i \in S, \eta_{i}+\lambda+\frac{\bar{\eta}_{i} q \alpha_{2}^{2} e^{\lambda \tau}}{\alpha_{1}^{2}} \leq \frac{\ln q}{\delta},
\end{aligned}
$$

then the zero solution of Equation (4.1) is exponentially stable in the mean square, where $\alpha_{1}=\min _{1 \leq i \leq N} \lambda_{\min }\left(Q_{i}\right), \alpha_{2}=\max _{1 \leq i \leq N} \lambda_{\max }\left(Q_{i}\right), q=\frac{1}{\max _{i \in S, 1 \leq k<+\infty}\left\{d_{i k}+\bar{d}_{i k} e^{\lambda \tau}\right\}}>1$.

Proof. We define $V \in \mathscr{C}^{2,1}\left(R^{n} \times[-\tau, \infty) \times S ; R^{+}\right)$by $V(x, t, i)=x^{T}(t) Q_{i} x(t)$. Clearly

$$
\alpha_{1}|x|^{2} \leq V(x, t, i) \leq \alpha_{2}|x|^{2} .
$$

Then for $t \in\left(t_{k-1}, t_{k}\right]$

$$
\begin{aligned}
& \mathscr{L} V(t, \phi)=2 \phi^{T}(0) Q_{i}\left[A_{i} \phi(0)+B_{i} \phi(-\tau(t))\right]+\left[C_{i} \phi(0)\right. \\
& \left.+D_{i} \phi(-\tau(t))\right]^{T} \times Q_{i}\left[C_{i} \phi(0)+D_{i} \phi(-\tau(t))\right]+\sum_{j=1}^{N} \gamma_{i j} \phi^{T}(0) Q_{j} \phi(0) \\
& =\phi^{T}(0)\left(Q_{i} A_{i}+A_{i}^{T} Q_{i}\right) \phi(0)+2 \phi^{T}(0) Q_{i} B_{i} \phi(-\tau(t)) \\
& +\phi^{T}(0) C_{i}^{T} Q_{i} C_{i} \phi(0)+\phi^{T}(0) C_{i}^{T} Q_{i} D_{i} \phi(-\tau(t))+\phi^{T}(-\tau(t)) D_{i}^{T} Q_{i} C_{i} \phi(0) \\
& +\phi^{T}(-\tau(t)) D_{i}^{T} Q_{i} D_{i} \phi(-\tau(t))+\sum_{j=1}^{N} \gamma_{i j} \phi^{T}(0) Q_{j} \phi(0) .
\end{aligned}
$$


In view of for any vectors $x, y \in R^{n}$, scalar $\varepsilon>0$, the following inequality holds

$$
2 x^{T} y \leq \varepsilon x^{T} x+\varepsilon^{-1} y^{T} y,
$$

then it follows that

$$
2 \phi^{T}(0) Q_{i} B_{i} \phi(-\tau(t)) \leq \rho_{i} \phi^{T}(0) Q_{i}^{2} \phi(0)+\rho_{i}^{-1} \phi(-\tau(t)) B_{i}^{T} B_{i} \phi(-\tau(t))
$$

and

$$
\begin{aligned}
& \phi^{T}(0) C_{i}^{T} Q_{i} D_{i} \phi(-\tau(t))+\phi^{T}(-\tau(t)) D_{i}^{T} Q_{i} C_{i} \phi(0) \\
& \quad \leq \sigma_{i} \phi^{T}(0) C_{i}^{T} C_{i} \phi(0)+\sigma_{i}^{-1} \phi^{T}(-\tau(t)) D_{i}^{T} Q_{i}^{2} D_{i} \phi(-\tau(t)) .
\end{aligned}
$$

Substituting (4.8) and (4.9) into (4.6) we can derive that

$$
\begin{aligned}
& \mathscr{L} V(\phi, t, i) \leq \phi^{T}(0)\left(Q_{i} A_{i}+A_{i}^{T} Q_{i}+\rho_{i} Q^{2}+C_{i}^{T} Q_{i} C_{i}+\sigma_{i} C_{i}^{T} C_{i}\right. \\
& \left.\quad+\sum_{j=1}^{N} \gamma_{i j} Q_{j}-\eta_{i} Q_{i}\right) \phi(0)+\phi^{T}(-\tau(t))\left(\rho_{i}^{-1} B_{i}^{T} B_{i}+\sigma_{i}^{-1} D_{i}^{T} Q_{i}^{2} D_{i}\right. \\
& \left.+D_{i}^{T} Q_{i} D_{i}-\bar{\eta}_{i} Q_{i}\right) \phi(-\tau(t))+\eta_{i} \phi^{T}(0) Q_{i} \phi(0)+\bar{\eta}_{i} \phi^{T}(-\tau(t)) Q_{i} \phi(-\tau(t)) \\
& \leq \eta_{i} \phi^{T}(0) Q_{i} \phi(0)+\bar{\eta}_{i} \phi^{T}(-\tau(t)) Q_{i} \phi(-\tau(t)) .
\end{aligned}
$$

Then

$$
E \mathscr{L} V(\phi, t, i)] \leq \eta_{i} E V(\phi(0), t, i)+\bar{\eta}_{i} \alpha_{2} E|\phi(-\tau(t))|^{2} .
$$

Next, if for $\forall \theta \in[-\tau, 0], E\left[\min _{1 \leq i \leq N} V(\phi(\theta), t, i)\right]<q e^{\lambda \tau} E\left[\max _{1 \leq i \leq N} V(\phi(\theta), t, i)\right]$, we have

$$
E|\phi(\theta)|^{2}<\frac{q \alpha_{2} e^{\lambda \tau}}{\alpha_{1}} E|\phi(0)|^{2}, \forall \theta \in[-\tau, 0] .
$$

Thus

$$
E[\mathscr{L} V(\phi, t, i)] \leq \eta_{i} E V(\phi(0), t, i)+\frac{\bar{\eta}_{i} q \alpha_{2}^{2} e^{\lambda \tau}}{\alpha_{1}} E|\phi(0)|^{2} \leq\left(\eta_{i}+\frac{\bar{\eta}_{i} q \alpha_{2}^{2} e^{\lambda \tau}}{\alpha_{1}^{2}}\right) E V(\phi(0), t, i) .
$$

For $t=t_{k}$, it follows from (ii) that

$$
\begin{aligned}
& E V\left(\phi(0)+I_{k}\left(\phi, \phi\left(-\tau\left(t_{k}\right)\right), t_{k}, i\right), t_{k}^{+}, i\right) \\
& \quad \leq d_{i k} E V\left(\phi(0), t_{k}, i\right)+d_{i k} E V\left(\phi\left(-\tau\left(t_{k}\right)\right), t_{k}-\tau\left(t_{k}\right), i\right)
\end{aligned}
$$

Consequently, the conclusions follow from Theorem 3.1. This completes the proof.

Theorem 4.2. Assume that exist symmetric positive definite matrices $Q_{i}$ and constants $\begin{gathered}d_{i k} \geq 0, \bar{d}_{i k} \geq 0, d_{i k}^{2}+\bar{d}_{i k}^{2} \neq 0, \varepsilon_{i}>0, \kappa_{i}>0, \zeta_{i}>0, \bar{\zeta}_{i} \geq 0, \mu>0, \lambda>\text { such } \\ 0, i \in S, k=1,2, \ldots\end{gathered}$ that (4.4) holds and the following conditions hold:

(i) for all $i \in S$

$$
\Theta_{i}=Q_{i} A_{i}+A_{i}^{T} Q_{i}+\varepsilon_{i} Q_{i}^{2}+C_{i}^{T} Q_{i} C_{i}+\kappa_{i} C_{i}^{T} C_{i}+\sum_{j=1}^{N} \gamma_{i j} Q_{j}+\zeta_{i} Q_{i} \leq 0
$$

and

$$
\bar{\Theta}_{i}=\varepsilon_{i}^{-1} B_{i}^{T} B_{i}+\kappa_{i}^{-1} D_{i}^{T} Q_{i}^{2} D_{i}+D_{i}^{T} Q_{i} D_{i}-\bar{\zeta}_{i} Q_{i} \leq 0 ;
$$


(ii) $\inf _{1 \leq k<+\infty}\left\{t_{k}-t_{k-1}\right\} \geq \mu$;

(iii) for all $i \in S, \zeta_{i}-\frac{\bar{\zeta}_{i} q \alpha_{2}^{2} e^{\lambda \tau}}{\alpha_{1}^{2}}-\lambda>\frac{\ln q}{\mu}$,

then the zero solution of Equation (4.1) is exponentially stable in the mean square, where $q=\max _{i \in S, 1 \leq k<+\infty}\left\{d_{i k}+\bar{d}_{i k} e^{\lambda \tau}\right\} \geq 1$.

Proof. By (iii), we choose sufficiently small $\varepsilon>0$ such that $\zeta_{i}-\frac{\bar{\zeta}_{i}(q+\varepsilon) \alpha_{2}^{2} e^{\lambda \tau}}{\alpha_{1}^{2}}-\lambda>\frac{\ln q}{\mu}$. We define

$V(x, t, i)=x^{T}(t) Q_{i} x(t)$. Similar to the proof of Theorem 4.1, we get for $t \in\left(t_{k-1}, t_{k}\right]$

$$
E \mathscr{L} V(\phi, t, i) \leq \zeta_{i} E V(\phi(0), t, i)+\bar{\zeta}_{i} \alpha_{2} E|\phi(-\tau(t))|^{2} .
$$

For $\forall \theta \in[-\tau, 0], E\left[\min _{1 \leq i \leq N} V(\phi(\theta), t, i)\right] \leq(q+\varepsilon) e^{\lambda \tau} E\left[\max _{1 \leq i \leq N} V(\phi(0), t, i)\right]$, we have

$$
E|\phi(\theta)|^{2} \leq \frac{(q+\varepsilon) \alpha_{2} e^{\lambda \tau}}{\alpha_{1}} E|\phi(0)|^{2} .
$$

Thus

$$
E[\mathscr{L} V(\phi, t, i)] \leq\left(-\zeta_{i}+\frac{\bar{\zeta}_{i}(q+\varepsilon) \alpha_{2}^{2} e^{\lambda \tau}}{\alpha_{1}^{2}}\right) E V(\phi(0), t, i) .
$$

Therefore, the conclusions follow from Theorem 3.2.

In the following, we shall establish tractable exponential stability conditions. To this end, we take

$$
\begin{aligned}
& Q_{i}=r_{i} I, \rho_{i}=\varepsilon_{i}=\frac{\left\|B_{i}\right\|}{r_{i}}, \sigma_{i}=\kappa_{i}=\frac{r_{i \|} D_{i} \|}{\left\|C_{i}\right\|}, \eta_{i}=\frac{1}{r_{i}}\left[\lambda_{\max }\left(r_{i} A_{i}+r_{i} A_{i}^{T}+\sum_{j=1}^{N} \gamma_{i j} \gamma_{j} I\right)+r_{i}\left\|B_{i}\right\|+r_{i}\left\|C_{i}\right\|^{2}+r_{i}\left\|C_{i}\right\|\left\|D_{i}\right\|\right], \\
& \zeta_{i}=-\frac{1}{r_{i}}\left[\lambda_{\max }\left(r_{i} A_{i}+r_{i} A_{i}^{T}+\sum_{j=1}^{N} \gamma_{i j} r_{j} I\right)+r_{i}\left\|B_{i}\right\|+r_{i}\left\|C_{i}\right\|^{2}+r_{i}\left\|C_{i}\right\|\left\|D_{i}\right\|\right], \bar{n}_{i}=\bar{\zeta}_{i}=\left\|B_{i}\right\|+\left\|C_{i}\right\|\left\|D_{i}\right\|+\left\|D_{i}\right\|^{2} .
\end{aligned}
$$

Corollary 4.1. Assume that there exist constants $d_{i k} \geq 0, \bar{d}_{i k} \geq 0, d_{i k}+\bar{d}_{i k} \neq 0, \delta>0, \lambda>0, r_{i}>0, i \in S, k=1,2, \ldots$ such that

(i) for all $i \in S$

$$
\begin{aligned}
& E\left[\left(x\left(t_{k}\right)+I_{k}\left(x\left(t_{k}\right), x\left(t_{k}-\tau\left(t_{k}\right)\right), t_{k}, i\right)\right)^{T}\left(x\left(t_{k}\right)+I_{k}\left(x\left(t_{k}\right), x\left(t_{k}-\tau\left(t_{k}\right)\right), t_{k}, i\right)\right)\right] \\
& \quad \leq d_{i k} E\left[x^{T}\left(t_{k}\right) x\left(t_{k}\right)\right]+\bar{d}_{i k} E\left[x^{T}\left(t_{k}-\tau\left(t_{k}\right)\right) x\left(t_{k}-\tau\left(t_{k}\right)\right)\right]
\end{aligned}
$$

(ii) $\sup _{1 \leq k<+\infty}\left\{t_{k}-t_{k-1}\right\} \leq \delta$;

(iii) for all $i \in S$

$$
\begin{aligned}
& \frac{1}{r_{i}}\left[\lambda \max \left(r_{i} A_{i}+r_{i} A_{i}^{T}+\sum_{j=1}^{N} \gamma_{i j} r_{j} I\right)+r_{i}\left\|B_{i}\right\|+r_{i}\left\|C_{i}\right\|^{2}+r_{i}\left\|C_{i}\right\|\left\|D_{i}\right\|\right] \\
& +\lambda+\frac{q \alpha_{2}^{2} e^{\lambda \tau}}{\alpha_{1}^{2}}\left[\left\|B_{i}\right\|+\left\|C_{i}\right\|\left\|D_{i}\right\|+\left\|D_{i}\right\|^{2}\right] \leq \frac{\ln q}{\delta},
\end{aligned}
$$

then the zero solution of Equation (4.1) is exponentially stable in the mean square, where $\alpha_{1}=\min _{1 \leq i \leq N} r_{i}, \alpha_{2}=\max _{1 \leq i \leq N} r_{i}, q=\frac{1}{\max _{i \in S, 1 \leq k<+\infty}\left\{d_{i k}+\bar{d}_{i k} e^{\lambda \tau}\right\}}>1$.

Corollary 4.2. Assume that there exist constants $d_{i k} \geq 0, \bar{d}_{i k} \geq 0, d_{i k}+\bar{d}_{i k} \neq 0, \mu>0, \lambda>0, r_{i}>0, i \in S, k=1,2, \ldots$ such that (4.20) holds and the following conditions are satisfied: 
(i) $\inf _{1 \leq k<+\infty}\left\{t_{k}-t_{k-1}\right\} \geq \mu$;

(ii) for all $i \in S$

$$
\begin{aligned}
& \frac{1}{r_{i}}\left[\lambda_{\max }\left(r_{i} A_{i}+r_{i} A_{i}^{T}+\sum_{j=1}^{N} \gamma_{i j} r_{j} I\right)+r_{i}\left\|B_{i}\right\|+r_{i}\left\|C_{i}\right\|^{2}+r_{i}\left\|C_{i}\right\|\left\|D_{i}\right\|\right] \\
& +\lambda+\frac{q \alpha_{2}^{2} e^{\lambda \tau}}{\alpha_{1}^{2}}\left[\left\|B_{i}\right\|+\left\|C_{i}\right\|\left\|D_{i}\right\|+\left\|D_{i}\right\|^{2}\right]+\frac{\ln q}{\mu}<0,
\end{aligned}
$$

then the zero solution of Equation (4.1) is exponentially stable in the mean square, where $q=\max _{i \in S, 1 \leq k<+\infty}\left\{d_{i k}+\bar{d}_{i k} e^{\lambda \tau}\right\} \geq 1$.

Next, we apply Corollaries 4.1 and 4.2 to establish some very useful criteria in terms of $M$ matrix which can be verified much more easily. If $A$ is a vector or matrix, by $A$ $\gg 0$ we mean all elements of $A$ are positive. If $A_{1}$ and $A_{2}$ are vectors or matrices with same dimensions we write $A_{1} \gg A_{2}$ if and only if $A_{1}-A_{2} \gg 0$. Moreover, we also adopt here the traditional natation by letting

$$
Z^{N \times N}=\left\{A=\left(a_{i j}\right)_{N \times N} \mid a_{i j} \leq 0, i \neq j\right\} .
$$

Definition 4.1. (see [12,28]) A square matrix $A=\left(a_{i j}\right)_{N \times N}$ is called a nonsingular $M$ matrix if $A$ can be expressed in the form $A=s I-B$ with $s>\rho(B)$ while all the elements of $B$ are nonnegative, where $I$ is the identity matrix and $\rho(B)$ the spectral radius of $B$.

Remark 4.1. If $A$ is a nonsingular $M$-matrix, then $A$ has nonpositive off-diagonal and positive diagonal entries, that is

$$
a_{i i}>0, \quad \text { while } a_{i j} \leq 0, \quad i \neq j .
$$

\section{Corollary $4.3 . \quad$ There exist constants} $d_{i k} \geq 0, \bar{d}_{i k} \geq 0, d_{i k}+\bar{d}_{i k} \neq 0, i \in S, k=1,2, \ldots$ such that (4.20) holds. If there exists $\lambda>0$ such that $\Xi-\Gamma$ is a nonsingular and for any $i \in S$

$$
r_{i}\left[\lambda+\frac{q \alpha_{2}^{2} e^{\lambda \tau}}{\alpha_{1}^{2}}\left(\left\|B_{i}\right\|+\left\|C_{i}\right\|\left\|D_{i}\right\|+\left\|D_{i}\right\|^{2}\right)\right] \leq 1,
$$

then the zero solution of Equation (4.1) is exponentially stable in the mean square, where $\Xi$ is the diagonal matrix

$$
\begin{aligned}
& \Xi=\operatorname{diag}\left[-\lambda_{\max }\left(A_{1}+A_{1}^{T}\right)-\left\|B_{1}\right\|-\left\|C_{1}\right\|^{2}-\left\|C_{1}\right\|\left\|D_{1}\right\|\right. \\
& \left.+\frac{\ln q}{\delta}, \ldots,-\lambda_{\max }\left(A_{N}+A_{N}^{T}\right)-\left\|B_{N}\right\|-\left\|C_{N}\right\|^{2}-\left\|C_{N}\right\|\left\|D_{N}\right\|+\frac{\ln q}{\delta}\right],
\end{aligned}
$$

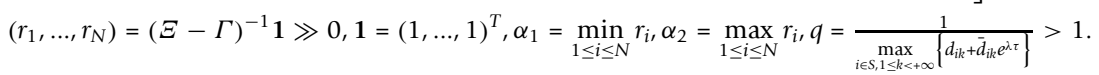

Proof. We conclude that all the elements of $(\Xi-\Gamma)^{-1}$ are nonnegative. So we have (4.24) holds, namely all $r_{i}$ are positive. Note

$$
(-\Xi+\Gamma) \mathbf{r}=-\mathbf{1},
$$

that is

$$
\lambda_{\max }\left(A_{i}+A_{i}^{T}\right) r_{i}+\sum_{j=1}^{N} \gamma_{i j} r_{j}+\left(\left\|B_{i}\right\|+\left\|C_{i}\right\|^{2}+\left\|C_{i}\right\|\left\|D_{i}\right\|-\frac{\ln q}{\delta}\right) r_{i}=-1 .
$$


Then we have for $i \in S$

$$
\begin{aligned}
& \frac{1}{r_{i}}\left[\lambda_{\max }\left(r_{i} A_{i}+r_{i} A_{i}+\sum_{j=1}^{N} \gamma_{i j} r_{j} I\right)+r_{i}\left\|B_{i}\right\|+r_{i}\left\|C_{i}\right\|^{2}\right. \\
& \left.\quad+r_{i}\left\|C_{i}\right\|\left\|D_{i}\right\|-\frac{r_{i} \ln q}{\delta}\right]+\frac{q \alpha_{2} e^{2 \tau \tau}}{\alpha_{12}}\left[\left\|B_{i}\right\|+\left\|C_{i}\right\|\left\|D_{i}\right\|+\left\|D_{i}\right\|^{2}\right] \\
& =\frac{1}{r_{i}}\left[\lambda_{\max }\left(r_{i} A_{i}+r_{i} A_{i^{T}}\right)+\sum_{j=1}^{N} \gamma_{i j} r_{j}+r_{i}\left\|B_{i}\right\|+r_{i}\left\|C_{i}\right\|^{2}\right. \\
& \left.\quad+r_{i}\left\|C_{i}\right\|\left\|D_{i}\right\|-\frac{r_{i} \ln q}{\delta}\right]+\lambda+\frac{q \alpha_{2} 2^{2 \tau}}{\alpha_{12}}\left(\left\|B_{i}\right\|+\left\|C_{i}\right\|\left\|D_{i}\right\|+\left\|D_{i}\right\|^{2}\right) \\
& =-\frac{1}{r_{i}}+\lambda+\frac{q \alpha_{2} 2^{2 e^{2} \tau}}{\alpha_{12}}\left(\left\|B_{i}\right\|+\left\|C_{i}\right\|\left\|D_{i}\right\|+\left\|D_{i}\right\|^{2}\right) \leq 0 .
\end{aligned}
$$

The required conclusion follows from Corollary 4.1.

Corollary 4.4. There exist constants $d_{i k} \geq 0, \bar{d}_{i k} \geq 0, d_{i k}+\bar{d}_{i k} \neq 0, i \in S, k=1,2, \ldots$ such that (4.17) holds. If there exists $\lambda>0$ such that $\bar{\Xi}-\Gamma$ is a nonsingular and for any $i \in S$

$$
r_{i}\left[\lambda+\frac{q \alpha_{2}^{2} e^{\lambda \tau}}{\alpha_{1}^{2}}\left(\left\|B_{i}\right\|+\left\|C_{i}\right\|\left\|D_{i}\right\|+\left\|D_{i}\right\|^{2}\right)\right]<1,
$$

then the zero solution of Equation (4.1) is exponentially stable in the mean square, where $\bar{\Xi}$ is the diagonal matrix denoted by

$$
\begin{gathered}
\bar{\Xi}=\operatorname{diag}\left[-\lambda_{\max }\left(A_{1}+A_{1}^{T}\right)-\left\|B_{1}\right\|-\left\|C_{1}\right\|^{2}-\left\|C_{1}\right\|\left\|D_{1}\right\|\right. \\
\left.-\frac{\ln q}{\mu}, \ldots,-\lambda_{\max }\left(A_{N}+A_{N}^{T}\right)-\left\|B_{N}\right\|-\left\|C_{N}\right\|^{2}-\left\|C_{N}\right\|\left\|D_{N}\right\|-\frac{\ln q}{\mu}\right], \\
\left(r_{1}, \ldots, r_{N}\right)=(\bar{\Xi}-\Gamma)^{-1} 1 \gg 0, \alpha_{1}=\min _{1 \leq i \leq N} r_{i}, \alpha_{2}=\max _{i \in S, 1 \leq i \leq N} r_{i,} q=\max _{1 \leq k<+\infty}\left\{d_{i k}+\bar{d}_{i k} e^{\lambda \tau}\right\} \geq 1 .
\end{gathered}
$$

\section{Examples and numerical simulations}

In this section, two examples are provided to illustrate our results.

Example 5.1. Let $\omega(t)$ be a scalar Brownian motion. Let $r(t), t \geq 0$ be a right-continuous Markov chain taking values in $S=\{1,2\}$ with the generator

$$
\left(\begin{array}{cc}
-3 & 3 \\
1 & -1
\end{array}\right)
$$

Consider the following scalar hybrid impulsive stochastic delay system

$$
\left\{\begin{array}{l}
d x(t)=\left[A_{r(t)} x(t)+B_{r(t)} x(t-1)\right] d t+\left[C_{r(t)} x(t)+D_{r(t)} x(t-1)\right] d \omega(t), \quad t \geq 0, \quad t \neq t_{k,} \\
\Delta x\left(t_{k}\right)=-0.6 x\left(t_{k}\right)+0.4 x\left(t_{k}-1\right), \quad k=1,2, \ldots,
\end{array}\right.
$$

where $t_{k}=0.003 k, \tau(t)=1, A_{1}=-1, A_{2}=-2, B_{1}=1, B_{2}=2, C_{1}=1, C_{2}=2, D_{1}=2$, $D_{2}=3, d_{1 k}=d_{2 k}=0.32, \bar{d}_{1 k}=\bar{d}_{2 k}=0.32$. Taking $r_{1}=r_{2}=1$, we see that there exists $\lambda>0$ such that

$$
\begin{aligned}
& 0.32+0.32 e^{\lambda}<1, \\
& \frac{1}{r_{1}}\left[\lambda_{\max }\left(r_{1} A_{1}+r_{1} A_{1}^{T}+\sum_{j=1}^{2} \gamma_{1 j} r_{j} I\right)+r_{1}\left\|B_{1}\right\|\right. \\
& \left.+r_{1}\left\|C_{1}\right\|^{2}+r_{1}\left\|C_{1}\right\|\left\|D_{1}\right\|\right]+\lambda+\frac{q \alpha_{2}^{2} e^{\lambda \tau}}{\alpha_{1}^{2}}\left[\left\|B_{1}\right\|+\left\|C_{1}\right\|\left\|D_{1}\right\|+\left\|D_{1}\right\|^{2}\right] \\
& =2+\lambda+\frac{7 e^{\lambda}}{0.32+0.32 e^{\lambda}} \leq-\frac{\ln \left(0.32+0.32 e^{\lambda}\right)}{0.003}
\end{aligned}
$$


and

$$
\begin{aligned}
\frac{1}{r_{2}} & {\left[\lambda_{\max }\left(r_{2} A_{2}+r_{2} A_{2}^{T}+\sum_{j=1}^{2} \gamma_{2 j} r_{j} I\right)+r_{2}\left\|B_{2}\right\|\right.} \\
& \left.+r_{2}\left\|C_{2}\right\|^{2}+r_{2}\left\|C_{2}\right\|\left\|D_{2}\right\|\right]+\lambda+\frac{q \alpha_{2}^{2} e^{\lambda \tau}}{\alpha_{1}^{2}}\left[\left\|B_{2}\right\|+\left\|C_{2}\right\|\left\|D_{2}\right\|+\left\|D_{2}\right\|^{2}\right] \\
& =8+\lambda+\frac{17 e^{\lambda}}{0.32+0.32 e^{\lambda}} \leq-\frac{\ln \left(0.32+032 e^{\lambda}\right)}{0003} .
\end{aligned}
$$

By Corollary 4.1, the zero solution of Equation (5.1) is exponentially stable in the mean square. Figure 1 depicts $x(t)$ of Equation (5.1) is exponentially stable in the mean square. Figure 2 depicts $x(t)$ of Equation (5.1) without delayed impulses.

Remark 5.1. From Figures 1 and 2, although hybrid stochastic delay system without impulses may be exponentially unstable in the mean square, adding delayed impulses may lead to exponentially stable in the mean square, which implies that impulses may change the stable behavior of an system.

Example 5.2. Let $r(t), t \geq 0$ be a right-continuous Markov chain taking values in $S=$ $\{1,2,3\}$ with the generator

$$
\left(\begin{array}{cc}
-2 & 2 \\
1 & -1
\end{array}\right) \text {. }
$$

Consider the following the 3-D hybrid impulsive stochastic delay system

$$
\left\{\begin{array}{l}
d x(t)=\left[A_{r(t)} x(t)+B_{r(t)} x(t-0.5)\right] d t+\left[C_{r(t)} x(t)+D_{r(t)} x(t-0.5)\right] d \omega(t), t \geq 0, t \neq t_{k}, \\
\Delta x\left(t_{k}\right)=-0.6 x\left(t_{k}\right)+0.4 x\left(t_{k}-0.5\right), k=1,2, \ldots .
\end{array}\right.
$$

where

$$
\begin{aligned}
& A_{1}=\left(\begin{array}{cc}
-1 & -1 \\
-0.5 & 0.4
\end{array}\right), A_{2}=\left(\begin{array}{cc}
-0.3 & 0.4 \\
0.2 & 0.1
\end{array}\right), B_{1}=\left(\begin{array}{ll}
0.6 & 0.8 \\
-1 & 0.6
\end{array}\right), B_{2}=\left(\begin{array}{cc}
-1 & 0.6 \\
0.7 & -0.2
\end{array}\right), \\
& C_{1}=\left(\begin{array}{cc}
0.2 & 0 \\
0 & 0.8
\end{array}\right), C_{2}=\left(\begin{array}{cc}
1 & 0 \\
0 & 0.6
\end{array}\right), D_{1}=\left(\begin{array}{cc}
0.7 & 0 \\
0 & 1
\end{array}\right), D_{2}=\left(\begin{array}{ll}
1 & 0 \\
0 & 1
\end{array}\right),
\end{aligned}
$$

$t_{k}=0.02 k, \tau(t)=0.5,\left\|B_{1}\right\|=1.1817,\left\|B_{2}\right\|=1.3650,\left\|C_{1}\right\|=0.8,\left\|C_{2}\right\|=1,\left\|D_{1}\right\|=$ $\left\|D_{2}\right\|=1$. It is easy to see that $d_{1 k}=d_{2 k}=0.20, \bar{d}_{1 k}=\bar{d}_{2 k}=\bar{d}_{3 k}=0.20$. By

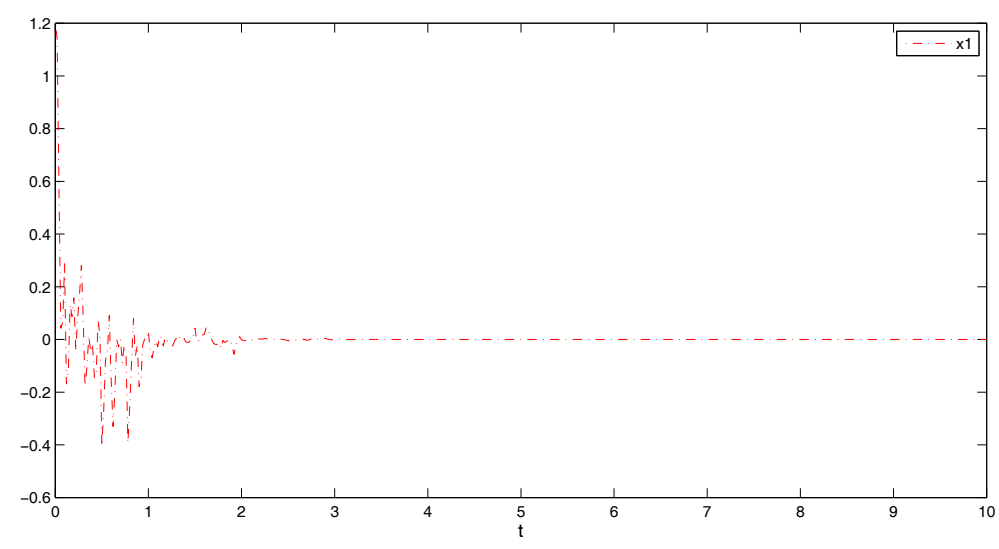

Figure 1 Trajectory of the states $x(t)$ of Equation (5.1) 


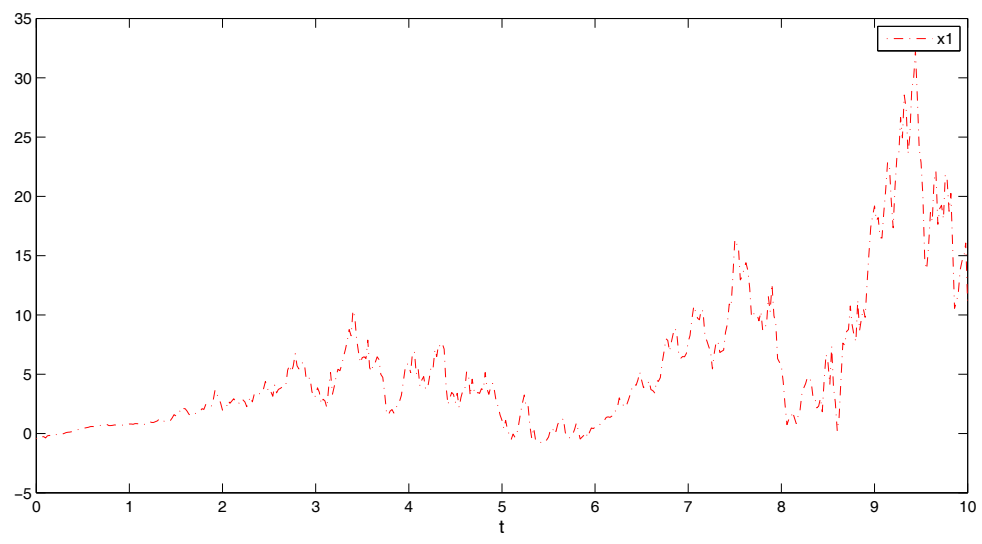

Figure 2 Trajectory of the states $x(t)$ of Equation (5.1) without delay impulses

computation, we have

$$
\lambda_{\text {max }}\left(A_{i}+A_{i}^{T}\right)=\left\{\begin{array}{l}
1.4518, i=1 \\
0.5211, i=2 .
\end{array}\right.
$$

Taking $\lambda=0.5$, we have

$$
\Xi=\operatorname{diag}[35.1065,35.2939] \text {. }
$$

Hence

$$
\Xi-\Gamma=\left(\begin{array}{cc}
16.0354 & -2 \\
-1 & 36.2939
\end{array}\right) \text {. }
$$

$\Xi-\Gamma$ is a nonsingular $M$-matrix. Then

$$
\left(r_{1}, r_{2}, r_{3}\right)^{T}=(\Xi-\Gamma)^{-1} \mathbf{1}=(0.0285,0.0284)^{T} \text {. }
$$

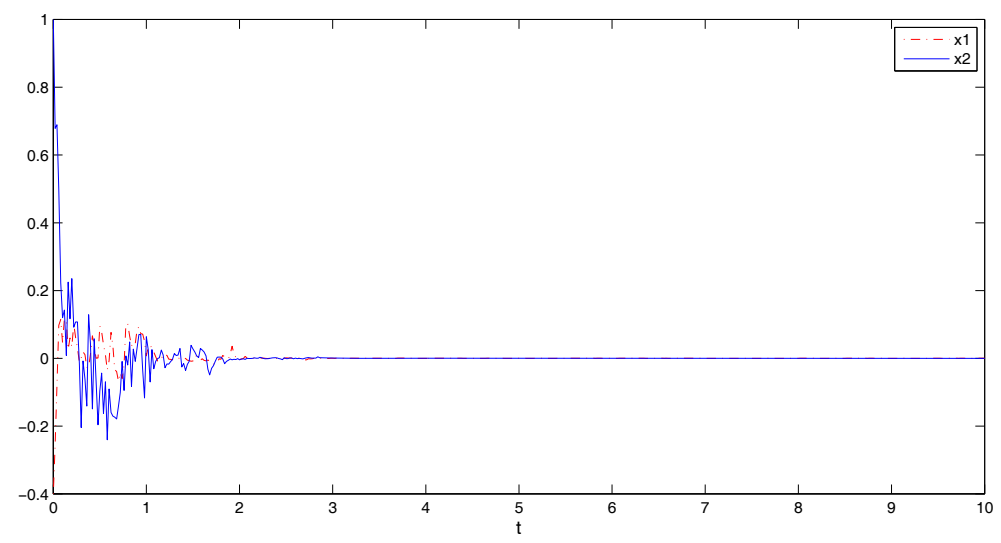

Figure 3 Trajectory of the states $x(t)$ of Equation (5.2) 


\section{Compute}

$$
r_{i}\left[0.5+\frac{\alpha_{2}^{2} \frac{1}{4}}{\alpha_{1}^{2}}\left(\left\|B_{i}\right\|+\left\|C_{i}\right\|\left\|D_{i}\right\|+\left\|D_{i}\right\|^{2}\right)\right] \leq 1, \quad i=1,2 .
$$

By Corollary 4.3, the zero solution of Equation (5.2) is exponentially stable in the mean square. Figure 3 depicts $x_{1}(t), x_{2}(t)$ of Equation (5.2) is exponentially stable in the mean square.

\section{Acknowledgements}

The authors would like to thank the referee and the associate editor for their very helpful suggestions. This work was supported by the National Natural Science Foundation of China under Grant No. 60874088, and the Natural Science Foundation of Jiangsu Province of China under Grant No. BK2009271, and JSPS Innovation Program under Grant No. CXZZ11_0132.

\section{Author details}

${ }^{1}$ Department of Mathematics, Southeast University, Nanjing 210096, P. R. China ${ }^{2}$ School of Mathematics, Jia Ying University, Meizhou Guangdong, 514015, P. R. China

\section{Authors' contributions}

All authors contributed equally to the manuscript. All authors read and approved the final manuscript.

\section{Competing interests}

The authors declare that they have no competing interests.

Received: 2 November 2011 Accepted: 14 May 2012 Published: 14 May 2012

\section{References}

1. Mao, X: Exponential Stability of Stochastic Differential Equations. Marcel Dekker, New York (1994)

2. Mao, X: Stochastic Differential Equations and Applications. Ellis Horwood, Chichester, UK (1997)

3. Karatzas, I, Shreve, SE: Brownian Motion and Stochastic Calculus. Springer-Verlag, Berlin (1991)

4. Chang, M: On Razumikhin-type stability conditions for stochastic functional differential equations. Math. Modell. 5 299-307 (1984). doi:10.1016/0270-0255(84)90007-1

5. Mao, X: Razumikhin type theorems on exponential stability of neutral stochastic functional differential equations. SIAM J. Math. Anal. 28(2):389-401 (1997). doi:10.1137/S0036141095290835

6. Janković, S, Randjelović, J, Jovanović, M: Razumikhin-type exponential stability criteria of neutral stochastic functional differential equations. J. Math. Anal. Appl. 355, 811-820 (2009). doi:10.1016/j.jmaa.2009.02.011

7. Ji, Y, Chizeck, HJ: Controllability, stabilizability and continuous-time Markovian jump linear quadratic control. IEEE Trans. Automat. Control. 35, 777-788 (1990). doi:10.1109/9.57016

8. Mariton, M: Jump Linear Systems in Automatic Control. Marcel Dekker, New York (1990)

9. Mao, X: Stability of stochastic differential equations with Markovian switching. Stochastic Process. Appl. 79, 45-67 (1999). doi:10.1016/S0304-4149(98)00070-2

10. Mao, X: Stochastic functional differential equations with Markovian switching. Funct. Diff. Equ. 6, 375-396 (1999)

11. Shaikhet, L: Stability of stochastic hereditary systems with Markov switching. Theory Stoch. Process. 2, 180-184 (1996)

12. Mao, X, Lam, J, Xu, S, Gao, H: Razumikhin method and exponential stability of hybrid stochastic delay interval systems. J. Math. Anal. Appl. 314, 45-66 (2006). doi:10.1016/j.jmaa.2005.03.056

13. Liu, B, Liu, X, Teo, K, Wang, Q: Razumikhin-type theorems on exponential stability of impulsive delay systems. IMA J. Appl. Math. 71, 47-61 (2006)

14. Anokhin, A, Berezansky, L, Braverman, E: Exponential stability of linear delay impulsive differential equations. J. Math. Anal. Appl. 193, 923-941 (1995). doi:10.1006/jmaa.1995.1275

15. Lakshmikantham, V, Bainov, DD, Simeonov, PS: Theory of Impulsive Differential Equations. World Scientific, Singapore (1989)

16. Samoilenko, AM, Perestyuk, NA: Impulsive Differential Equations. World Scientific, Singapore (1995)

17. Shen, J, Yan, J: Razumikhin type stability theorems for impulsive functional differential equations. Nonlinear Anal. 33 519-531 (1998). doi:10.1016/S0362-546X(97)00565-8

18. Liu, XZ, Wang, Q: The method of Lyapunov functionals and exponential stability of impulsive systems with time delay. Nonlinear Anal. 66(7):1465-1484 (2007). doi:10.1016/..na.2006.02.004

19. Wu, QJ, Zhou, J, Xiang, L: Global exponential stability of impulsive differential equations with any time delays. Appl. Math. Lett. 23, 143-147 (2010). doi:10.1016/j.aml.2009.09.001

20. Pan, LJ, Cao, JD: Exponential stability of impulsive stochastic functional differential equations. J. Math. Anal. Appl. 382, 672-685 (2011). doi:10.1016/j.jmaa.2011.04.084

21. Liu, B: Stability of solutions for stochastic impulsive systems via comparison approach. IEEE Trans. Automat. Control. 53, 2128-2133 (2008)

22. Peng, SG, Zhang, Y: Razumikhin-type theorems on pth moment exponential stability of impulsive stochastic delay differential equations. IEEE Trans. Automat. Control. 55, 1917-1922 (2010) 
23. Cheng, P, Deng, FQ: Global exponential stability of impulsive stochastic functional differential systems. Stat. Probab. Lett. 80, 1854-1862 (2010). doi:10.1016/j.spl.2010.08.011

24. Sakthivel, R, Luo, J: Asymptotic stability of impulsive stochastic partial differential equations with infinite delays. J. Math. Anal. Appl. 356, 1-6 (2009). doi:10.1016/j.jmaa.2009.02.002

25. Anguraj, A, Vinodkumar, A: Existence, uniqueness and stability results of random impulsive semilinear differential systems. Nonlinear Anal Hybr. Syst. 4, 475-483 (2010). doi:10.1016/j.nahs.2009.11.004

26. Li, CX, Sun, JT: Stability analysis of nonlinear stochastic differential delay systems under impulsive control. Phys. Lett. A. 374, 1154-1158 (2010). doi:10.1016/j.physleta.2009.12.065

27. $\mathrm{Wu}, \mathrm{HJ}$, Sun, JT: $p$-moment stability of Stochastic differential equations with impulsive jump and Markovian switching. Automatica. 42, 1753-1759 (2006). doi:10.1016/j.automatica.2006.05.009

28. Berman, A, Plemmons, RJ: Nonnegative Matrices in the Mathematical Sciences. SIAM, Philadelphia (1994)

doi:10.1186/1687-1847-2012-61

Cite this article as: Pan and Cao: Exponential stability of stochastic functional differential equations with

Markovian switching and delayed impulses via Razumikhin method. Advances in Difference Equations 2012 2012:61.

\section{Submit your manuscript to a SpringerOpen ${ }^{\ominus}$} journal and benefit from:

- Convenient online submission

- Rigorous peer review

- Immediate publication on acceptance

- Open access: articles freely available online

- High visibility within the field

- Retaining the copyright to your article

Submit your next manuscript at $\gg$ springeropen.com 